\title{
Herausforderungen und Gestaltungschancen aufgrund von MiFID und EU-Vermittlerrichtlinie in der Kundenberatung
}

von

Hans Ulrich Buhl, Marcus Kaiser

in: Zeitschrift für Bankrecht und Bankwirtschaft 20 (2008) 1, S. 43-51 


\section{Hans Ulrich Buhl, Marcus Kaiser}

\section{Herausforderungen und Gestaltungschancen aufgrund von MiFID und EU-Vermittlerrichtlinie in der Kundenberatung}

Die neuen EU-Richtlinien MiFID sowie zur Versicherungsvermittlung haben zum Ziel, den Verbraucherschutz im Bereich der Anlageberatung bei Finanzdienstleistungen zu stärken. Neben weiteren Maßnahmen schreibt der Gesetzgeber implizit auch die Dokumentation von personenbezogenen Kundendaten vor, wodurch der Datenschutz auf den Plan tritt. Um der Anforderung der Richtlinien nach einer besseren Individualisierung der Finanzdienstleistung auch im Massenkundengeschäft nachkommen zu können, sind Banken und Versicherungen auf die Unterstützung durch Informationstechnologie angewiesen. Daher wird im Beitrag ein wissenschaftlich fundiertes Konzept zur individualisierten Anlageberatung im Finanzdienstleistungsbereich vorgestellt, welches auch erfolgreich in die Praxis transferiert wurde

\section{Einleitung}

Die einschlägige Literatur diskutiert seit mehreren Jahren, welche Strategieoptionen Banken und Versicherungen haben. ${ }^{1}$ Dabei scheint es letzten Endes auf die Erkenntnis von Porter ${ }^{2}$ hinauszulaufen: Finanzdienstleister können zwischen Kostenführerschaft und Differenzierung wählen. ${ }^{3}$

Verfolgt ein Unternehmen die Strategie der Kostenführerschaft, so versucht es, seine Produkte zu den geringst möglichen Kosten herzustellen, um zum günstigsten Preis anbieten zu können. ${ }^{4}$ In vielen Fällen geht eine möglichst kostengünstige Produktion mit einer hohen Standardisierung der Produkte einher, weswegen diese häufig kaum angepasst werden können. Finanzdienstleistungen sind Informationsgüter und daher teuer herzustellen, aber billig zu kopieren, weswegen sie leicht von Konkurrenten imitiert werden können. ${ }^{5}$ Somit sind standardisierte Finanzdienstleistungen in einem hohen Maße austauschbar mit der Folge

\footnotetext{
${ }^{1}$ Vgl. Hedley et al., 2006, Jagersma, 2006, Kundisch, 2003, Nellis et al., 2000, Sherwood, 1995.

${ }^{2}$ Vgl. Porter, 1998.

${ }^{3}$ Vgl. Buhl et al., 2002.

${ }^{4}$ Vgl. Porter, 1998, S. 12.

${ }^{5}$ Vgl. Shapiro/Varian, 1998.
} 
geringer Wechselbarrieren. Daher kann ein rationaler Kunde leicht zu einem anderen Finanzdienstleister wechseln, falls dieser die gleiche Finanzdienstleistungen zu einem geringeren Preis anbietet. Aus wirtschaftstheoretischer Sicht kann nur derjenige Anbieter, der den geringsten Preis anbietet, auf solchen Märkten überleben. Auf dem realen Markt für Finanzdienstleistungen werden zwar mehr als einer, aber nur einige wenige Kostenführer langfristig bestehen. Zudem ist Kostenführerschaft eine riskante Strategie für Finanzdienstleister mit hohen Fixkosten (z. B. seit langem etablierte Allfinanzdienstleister mit einem dichten Netz von Bankfilialen), da diese preislich nur schwer mit Konkurrenten mithalten können, die hauptsächlich zu niedrigen Fixkosten anbieten (z. B. über Internet oder Call-Center). Daher kommt die Literatur in der Regel zum Schluss, dass Kostenführerschaft für die meisten Finanzdienstleister keine geeignete Strategie ist. ${ }^{6}$

Demgegenüber zielt die Differenzierung darauf ab, ein Angebot zu erstellen, das der Kunde als einzigartig wahrnimmt. ${ }^{7}$ Wie bereits erwähnt, können Finanzdienstleistungen leicht kopiert werden. Daher muss ein Finanzdienstleister für das einzigartige Problem des Kunden eine einzigartige Lösung generieren, die von seinen Bedürfnissen, Wünschen und Zielen bestimmt wird. Zudem müssen dabei die gegenwärtige Rolle des Kunden und seine spezifische Situation berücksichtigt werden, kurz: Die Lösung muss individualisiert werden. Bietet ein Finanzdienstleister derartige - auf den Kunden zugeschnittene - Lösungen an, bringt ihm dies zwei Vorteile: Zum einen entzieht er sich damit dem Wettbewerb, da sein Angebot nur schwer vergleichbar und somit kaum austauschbar ist. Zum anderen erzeugt die Generierung von individualisierten Lösungen auf beiden Seiten, der des Kunden und der des Finanzdienstleisters, nicht unerheblichen Aufwand. Wird dieser Aufwand durch eine hohe Qualität der Anlageberatungsleistung (was mit der Generierung einer individualisierten Lösung gleichgesetzt werden kann), entlohnt, steigt das Vertrauen des Kunden in den Finanzdienstleister. In der Folge steigen Loyalität und Kundenbindung. ${ }^{8}$

\section{Status quo der Anlageberatung}

Die obige Diskussion zeigt auf, dass für die meisten Finanzdienstleister Differenzierung die vielversprechendere Strategie darstellt. In der Tat behaupten viele Banken und Versicherungen, diese Strategie zu verfolgen: Gemäß einer Studie von Steria Mummert

\footnotetext{
${ }^{6}$ Vgl. Buhl et al., 2002.

${ }^{7}$ Vgl. Porter, 1998, S. 14.

${ }^{8}$ Vgl. Fridgen/Steck, 2002.
} 
Consulting wollen 90\% der Banken ihre Vertriebsprozesse und -software anpassen, um eine bessere Kundenorientierung zu erreichen. ${ }^{9}$ Bei Finanzdienstleistungen ist die Umsetzung der Kundenorientierung eng verknüpft mit dem Angebot einer qualitativ hochwertigen Anlageberatung.

Betrachtet man den gegenwärtigen Zustand der Anlageberatung, fällt einem auf, dass die unterschiedlichen Akteure diesen unterschiedlich bewerten. Vertreter von Banken und Versicherungen erachten die Qualität der Anlageberatung als gut. So titelt der Bundesverband Deutscher Banken: „Gut beraten - Neun von zehn Kunden sind mit ihrer Bank zufrieden”. ${ }^{10}$ K.-P. Schackmann-Fallis vom Deutschen Sparkassen- und Giroverband wird wie folgt zitiert: „Die Qualität der Kundenaufklärung und -beratung bewegt sich bereits jetzt auf hohem Niveau.“" Demgegenüber zeigen sowohl wissenschaftliche Untersuchungen als auch populärwissenschaftliche Studien, dass die Qualität der Anlageberatung verbesserungsbedürftig ist. ${ }^{12}$ Das jüngste Beispiel ist ein Bericht der Zeitschrift Finanztest über Beratungen für Privatkredite: In vielen Fällen waren die Empfehlungen für das Problem des Kunden nicht geeignet, nicht genügend auf seine Situation zugeschnitten oder dienten einfach nur der Zielsetzung, die Provision für den Finanzdienstleister und den Berater zu maximieren. Einige Berater verstießen sogar gegen geltendes Recht, indem sie ohne Erlaubnis des Kunden bei der SCHUFA Auskunft über dessen Kreditwürdigkeit einholten. ${ }^{13}$ Die schlechte Qualität liegt oftmals auch an der Qualifikation der Berater: So ist deren Leistung einer Studie zufolge in jedem zweiten Fall nur mittelmäßig und ca. in jedem dritten Fall schwach. ${ }^{14}$ V. a. im Massenkundengeschäft kann in vielen Fällen von einem „systematisch koordinierten Planungsprozess“ - wie die Finanzdienstleistungsliteratur die Anlageberatung definiert $^{15}$ - kaum die Rede sein. Stattdessen wird häufig beklagt, dass die vorherrschende Entlohnungspraxis auf Umsatzbasis den provisionsgetriebenen Produktverkauf fördert, aber „kaum Anreize für eine qualitativ hochwertige Anlageberatung [bietet].“16 Vor diesem

\footnotetext{
${ }^{9}$ Vgl. Diegelmann/Deter, 2006.

${ }^{10}$ Vgl. Bundesverband Deutscher Banken, 2007.

${ }^{11}$ Siehe Steevens, 2007.

${ }^{12}$ Vgl. Booz Allen Hamilton GmbH, 2007, Hetzer/Palan, 2007, Klöckner, 2007, Oberhuber, 2007.

${ }^{13}$ Vgl. Stiftung Warentest, 2007.

${ }^{14}$ Vgl. Schmidt-Gallas et al., 2007.

${ }^{15}$ Siehe Tilmes, 2001.

${ }^{16}$ Siehe Kaas/Severidt, 2002.
} 
Hintergrund erließ der europäische Gesetzgeber zwei Richtlinien, die u.a. eine Verbesserung des Verbraucherschutzes in der privaten Finanzplanung zum Ziel haben.

\section{Versicherungsvermittlerrichtlinie und MiFID}

Die „Richtlinie des Europäischen Parlaments und des Rates über Versicherungsvermittlung“ wurde am 09.12.2002 verabschiedet. Das „Gesetz zur Neuregelung des Versicherungsvermittlerrechts“ setzt diese in deutsches Recht um und ist zum 22.05.2007 in Kraft getreten. Dieses bezeichnen wir im Folgenden mit Versicherungsvermittlerrichtlinie (VVR), von der in Deutschland ca. 500.000 Vermittler betroffen sind. ${ }^{17}$ MiFID (engl. Markets in Financial Instruments Directive) steht für das „Finanzmarkt-Richtlinie-Umsetzungsgesetz“, das in Deutschland die „EU-Richtlinie über Märkte für Finanzinstrumente“ in nationales Recht umsetzt. Das Gesetzgebungsverfahren in Deutschland wurde im Mai 2007 abgeschlossen, das Gesetz trat zum 01.11.2007 in Kraft.

Abbildung 1 zeigt ausgewählte Maßnahmen, welche die beiden Richtlinien vorsehen. Zu den Maßnahmen, die beide Richtlinien enthalten, gehören die Beratungs- und Dokumentationspflichten, welche im Folgenden Schwerpunkt dieser Arbeit sein werden.

Abbildung 1: Ausgewählte Maßnahmen der EU-Vermittlerrichtlinie sowie der MiFID

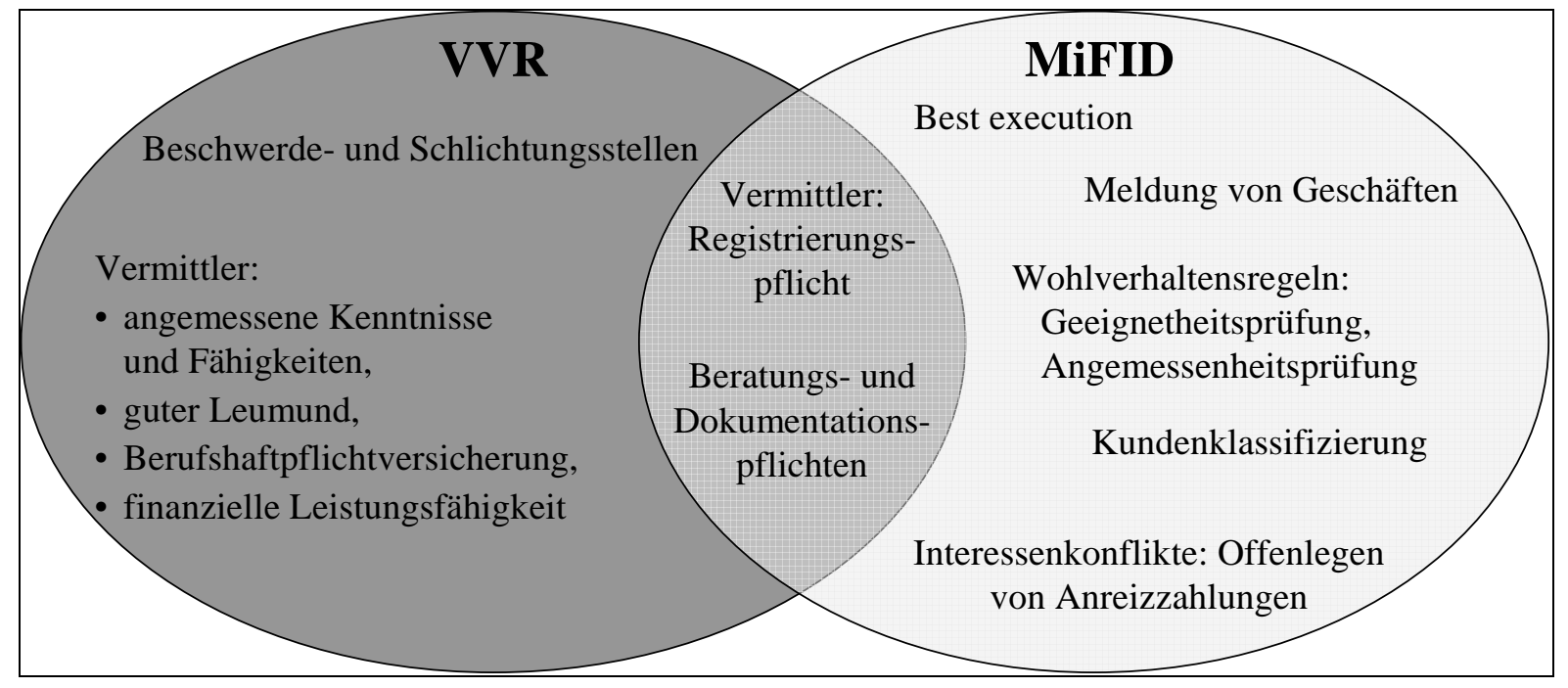

Beide Richtlinien schreiben also einen in der Theorie eigentlich obligatorischen, in der Praxis aber offensichtlich unzureichend durchgeführten, Vorgang als verpflichtend vor: Die Dokumentation der einzelnen Prozessschritte der Anlageberatung. Eine Anlageberatung von hoher Qualität ist auf Kundendaten angewiesen: Sie bilden die Grundlage, um eine

\footnotetext{
${ }^{17}$ Vgl. Maschmeyer, 2007.
} 
individuelle Lösung für des Kunden Problem zu finden. ${ }^{18}$ In einer individualisierten Anlageberatung werden Kundendaten als Eingangsparameter für Regeln, Berechungen und Simulationen verwendet, um die beste Lösung für den Kunden zu identifizieren. Im Folgenden wird analysiert, welche Kundendaten aufgrund der beiden Richtlinien dokumentiert werden müssen.

\section{Dokumentationspflicht}

\section{IV.1 Versicherungsvermittlerrichtlinie}

Die VVR schreibt vor, die Wünsche und Bedürfnisse des Kunden sowie die Gründe für den erteilten Rat festzustellen, zu dokumentieren und die Dokumentation an den Kunden auszuhändigen. Im Falle von Beratungsfehlern, die auf eine Verletzung dieser Verpflichtungen zurückzuführen sind, hat der Kunde Anspruch auf Schadensersatz durch den Berater. Da der Gesetzgeber weder bei den Wünschen und Bedürfnissen des Kunden noch bei den Gründen für den erteilten Rat exakt vorschreibt, wie diese zu dokumentieren sind, ist nicht auf den ersten Blick ersichtlich, welche Daten zu dokumentieren sind. Dass es sich aber bei den zu dokumentierenden Informationen in den meisten Fällen um personenbezogene Daten handelt, soll das folgende Beispiel verdeutlichen:

Ein Kunde sucht seinen Berater auf, da er für seine Mietwohnung eine Hausratversicherung abschließen möchte. Eine Faustregel besagt, dass die Versicherungssumme mit Hilfe der Formel Fläche der Wohnung(in $\left.\mathrm{m}^{2}\right)$ x 650 EUR festzulegen ist. Diese Formel genügt nicht den Ansprüchen einer Individualisierung, da es viele Fälle gibt, in denen ihre Anwendung zu einer Unter- bzw. Überdeckung führt. ${ }^{19}$ Dennoch soll sie hier zur Veranschaulichung vereinfachend als geeignet angenommen werden. Dann muss der Berater die Formel (als Grundlage für die Bedürfnisermittlung) und das in sie eingehende personenbezogene Datum (die $\mathrm{m}^{2}$-Zahl der Wohnung des Kunden) der Logik der VVR folgend als Begründung dokumentieren.

Bereits dieses einfache Beispiel veranschaulicht, dass bei der Dokumentation sowohl der Ermittlung der Wünsche und Bedürfnisse als auch der Begründung des erteilten Rats personenbezogene Daten verarbeitet werden. In der Realität gestaltet sich eine Individualisierung wesentlich komplexer. Dies verdeutlichen die von verschiedenen

\footnotetext{
${ }^{18}$ Vgl. Böckhoff/Stracke, 2003, Harrison, 2005, Kruschev, 1999, Schmidt, 2005, Tilmes, 2001.

${ }^{19}$ Vgl. Bund der Versicherten, 2007.
} 
Organisationen erarbeiteten Vorlagen zur Dokumentation: Für den im Beispiel betrachteten Fall einer Hausratversicherung wird z. B. empfohlen, über 25 Angaben des Kunden einzuholen und zu dokumentieren (z. B. „War Ihr Hausrat in den letzten fünf Jahren von Schäden betroffen?“, „Ist die Wohnung länger als 60 Tage ununterbrochen unbewohnt?““20$)$.

\section{IV.2 Richtlinie über Märkte für Finanzinstrumente}

Nicht nur im Bereich der Versicherungen, auch bei Wertpapieren zielt der Gesetzgeber auf eine Verbesserung des Verbraucherschutzes ab. Die MiFID definiert drei Dienstleistungstypen (Portfolioverwaltung und Anlageberatung; Beratungsfreie Finanzdienstleistungen; Reines Ausführungsgeschäft) ${ }^{21}$, für die sie vorschreibt: „Eine Wertpapierfirma sorgt dafür, dass Aufzeichnungen über alle ihre Dienstleistungen und Geschäfte geführt werden, die ausreichen, um der zuständigen Behörde zu ermöglichen, die Einhaltung der Anforderungen dieser Richtlinie zu überprüfen und sich vor allem zu vergewissern, dass die Wertpapierfirma sämtlichen Verpflichtungen gegenüber den Kunden oder potenziellen Kunden nachgekommen ist.“ Unter diese Dokumentationspflicht fällt auch die Anforderung der MiFID, dass Berater bei der Anlageberatung Informationen über die Kenntnisse und Erfahrungen des Kunden sowie über seine finanziellen Verhältnisse, Verpflichtungen und Anlageziele einholen müssen. ${ }^{22}$ Auch wenn bislang für die MiFID noch keine Dokumentationsempfehlungen erarbeitet wurden, ist abzusehen, dass zur Erfüllung dieser Anforderungen personenbezogene Daten des Kunden zu verarbeiten sind.

\section{IV.3 Datenschutz}

Aus den obigen Ausführungen wird deutlich, dass es sich bei den gemäß den beiden Richtlinien zu dokumentierenden Informationen i.d.R. um personenbezogene Kundendaten handelt. Dies ist insofern von Bedeutung, als hierdurch der Datenschutz auf den Plan tritt. Speziell im Massenkundengeschäft erlaubt es erst der Einsatz von Informationssystemen, individualisierte Anlageberatung wirtschaftlich anzubieten. Da hierbei - wie oben dargestellt - personenbezogene Daten automatisiert verarbeitet werden, ist eine Auseinandersetzung mit

\footnotetext{
${ }^{20}$ Siehe Arbeitskreis EU-Vermittlerrichtlinie Dokumentation, 2006.

${ }^{21}$ Siehe Duve/Keller, 2006.

${ }^{22}$ Vgl. Balzer, 2007, Duve/Keller, 2006.
} 
den datenschutzrechtlichen Bedingungen im Bereich der individualisierten Anlageberatung notwendig.

Der Datenschutz erachtet die Verarbeitung von personenbezogenen Daten nur dann als zulässig, wenn der Gesetzgeber oder der Betroffene diese hinsichtlich Umfang und Zweck gebilligt hat. ${ }^{23}$ Für die private Finanzplanung existieren keine speziellen Vorschriften, mit denen der Gesetzgeber die Verarbeitung explizit erlauben würde. Wie jedoch die obigen Beispiel illustrieren, wird durch die Dokumentationspflicht de facto eine solche Verarbeitung gefordert.

Die VVR problematisiert die Tatsache, dass die Dokumentation im Widerspruch zum Datenschutz steht, nicht. Daher ist den Beratern dieser Widerspruch oft nicht bewusst, er wird in der praxisorientierten Literatur nach Kenntnis des Autors nur am Rande angesprochen. ${ }^{24}$ Zudem macht die VVR keine konkreten Vorgaben bzgl. der tatsächlich abzufragenden personenbezogenen Daten. Somit werden die vom Gesetzgeber im Unklaren gelassenen Berater, die sich vor Schadensersatzforderungen schützen wollen, im Zweifelsfall eher zu viele als zu wenige Daten abfragen und dokumentieren. Dies steht wiederum im Widerspruch zu den im Datenschutz geltenden Prinzipien der Datenvermeidung bzw. der Datensparsamkeit.

Aber nicht nur der Berater, auch der Kunde gerät in ein Dilemma: Ist ihm daran gelegen, seine Daten nicht für die Dokumentation preiszugeben, muss er nicht nur auf eine qualitativ hochwertige Beratung verzichten, da eine solche auf eine gute Datenbasis angewiesen ist. Zudem muss der Kunde gemäß VVR eine Erklärung unterzeichnen, die ihn ausdrücklich darauf hinweist, dass er mit seiner Unterschrift auf Schadensersatzansprüche gegenüber dem Berater verzichtet. Bei der Abwägung zwischen den beiden Möglichkeiten werden sich Kunden in vielen Fällen gegen ihr Recht auf Datenschutz und zugunsten des Schadensersatzanspruchs bei einer Falschberatung entscheiden, ein Verhalten, das auch die Stiftung Warentest empfiehlt. ${ }^{25}$ Auch Vertreter der Versicherungspraxis raten Beratern, die Verzichtserklärung nur in Ausnahmefällen einzusetzen. ${ }^{26}$

Bei der MiFID muss der Kunde nicht auf seinen Schadensersatzanspruch verzichten, denn diese gewährt ihm einen solchen nicht explizit und sieht daher auch keine Möglichkeit zum

\footnotetext{
${ }^{23}$ Vgl. Roßnagel, 2005.

${ }^{24}$ Vgl. Warth/Beenken, 2005.

${ }^{25}$ Vgl. Stiftung Warentest, 2006.

${ }^{26}$ Vgl. Fiala, 2007.
} 
Verzicht auf Schadensersatz vor. Möchte der Kunde aber seine Daten nicht zur Dokumentation weitergeben, so darf der Berater ihm bei bestimmten Dienstleistungstypen keine Empfehlung aussprechen. Des weiteren ist bei der MiFID datenschutzrechtlich bedenklich, dass die Dokumentation nicht - wie bei der VVR - vom Kunden unterschrieben und an diesen ausgehändigt werden muss. Stattdessen dient sie lediglich der Überprüfung der Dokumentationspflicht durch die zuständige Behörde. Dies stellt eine Datenschutz-rechtlich eigentlich problematische Weitergabe an Dritte dar. Verbraucherschutzrechtlich ist hieran kritisch, dass die Beweislast im Falle von Streitigkeiten beim Kunden bleibt.

Zusammenfassend lässt sich sagen, dass die MiFID mit dem Datenschutz in ähnlicher Weise konfligiert wie die VVR, ohne dass dies in einer der beiden Vorschriften thematisiert wird. Es kann nicht davon ausgegangen werden, dass für VVR und MiFID der Datenschutz keine Rolle spielt, da eine Einschränkung des Datenschutzes einer expliziten Rechtsvorschrift bedarf, ${ }^{27}$ die in beiden Fällen nicht gegeben ist. Der Gesetzgeber ist aufgefordert, die Widersprüche, die er hier schafft, zu klären. In der Praxis zeichnet sich folgendes Vorgehen ab: Datenschutz, VVR und MiFID werden als regulatorische Pflicht aufgefasst, die es zu erfüllen gilt. Hierauf weist die Tatsache hin, dass die Vorlagen zur Dokumentation nach VVR eine vom Kunden zu unterzeichnende Datenschutzerklärung enthalten.

\section{IV.4 Folgen}

Vertreter der Praxis äußern neben den hohen Kosten, welche die Umstellungen aufgrund der beiden EU-Richtlinien mit sich bringen, auch Bedenken dahingehend, dass die Dokumentationspflicht zu einem Rückgang des Absatzes führen wird. Neben einer zu erwartenden höheren Beratungsqualität und damit einhergehenden gesteigerten Kundenzufriedenheit bieten sich jedoch für die Unternehmen weitere Nutzenpotenziale. Zunächst lässt sich ein verbessertes Cross-Selling nennen, da Finanzdienstleister ihre Kunden aufgrund der dokumentierten Daten gezielter auf einen bestimmten Bedarf ansprechen können. Zudem müssen für die Umsetzung der Richtlinien ohnehin Projekte initiiert und der damit verbundene Aufwand geleistet werden. Da es für die Erstellung einer Dokumentation notwendig ist, alle Kundendaten jederzeit korrekt vorzuhalten, ist die heutzutage häufig vorzufindende, historisch gewachsene, verteilte sowie redundante Kundendatenhaltung mit der Folge von inkonsistenten und fehlerhaften Daten kritisch. Die Unternehmen könnten die

\footnotetext{
${ }^{27}$ Vgl. Yildirim, 2004.
} 
angesprochenen Rüstkosten nutzen und die Zielsetzung derartiger Projekte dahingehend erweitern, dass ein Schritt in Richtung kundenzentrierter Informationssysteme erfolgt, um die Kundendaten redundanzfrei in einer auf logischer Ebene einheitlichen Kundendatenbank abzulegen. Aber auch wenn nur die Mindestanforderungen aus den EU-Richtlinien erfüllt werden, profitieren Unternehmen mit deren Umsetzung durch eine höhere Datenqualität. So zeigen Heinrich et al. $^{28}$ auf Basis von Auswertungen bei einem großen deutschen Versicherungsunternehmen, dass diejenigen Vertriebsagenturen, die mehr Daten über den Kunden dokumentieren, auch erfolgreicher sind: Sie erzielten im Durchschnitt deutlich höhere Umsätze als diejenigen Agenturen, die weniger Kundendaten dokumentieren. Dieser Zusammenhang gilt jedoch nicht pauschal, sondern für unterschiedliche Arten von Daten in unterschiedlichem Ausmaße. Daher ist eine detaillierte Untersuchung notwendig. Eine höhere Datenqualität kann zudem - unter Berücksichtigung der datenschutzrechtlichen Vorschriften - z. B. zu höheren Erfolgsquoten im Kampagnenmanagement oder zu einer genaueren Abschätzung der Potenziale einzelner Kunden oder Kundengruppen führen. Finanzdienstleister sollten also nicht nur den Aufwand der regulatorischen Anforderungen sehen, sondern die dahinter stehenden Kundenbedürfnisse sowie die sich hieraus ergebenden Chancen erkennen.

\section{Individualisierung der Anlageberatung}

Einerseits ist die im vergangenen Abschnitt beschriebene Dokumentationspflicht in beiden Richtlinien eine von mehreren Schutzmaßnahmen, um eine verbesserte Individualisierung der Anlageberatung zu erreichen. Beide Richtlinien sehen dabei tendenziell alle Privatkunden als schutzbedürftige, unmündige Akteure an, welche die Qualität der Beratungsleistung durch den Finanzdienstleister nicht beurteilen können. Andererseits wird von Finanzdienstleistern verlangt, die Lösungen an die Eigenschaften, Bedürfnisse und Kenntnisse des jeweiligen Kunden sowie an seine spezielle Situation und Rolle anzupassen. ${ }^{29}$ Hierzu ist anzumerken, dass ein Anbieter die vorgesehenen Schutzmaßnahmen umso weniger fürchten muss, je qualitativ hochwertiger und damit individualisierter seine Anlageberatung ist. Neben den in der Einleitung dargelegten Gründen ist dies ein weiterer Grund dafür, warum Finanzdienstleister die Individualisierung ihrer Produkte vorantreiben sollten.

\footnotetext{
${ }^{28}$ Heinrich et al., 2007.

${ }^{29}$ Vgl. Mertens/Griese, 2002.
} 
Gleichzeitig sind die Bedenken der Branche hinsichtlich der Kosten, die mit den Dokumentationspflichten im speziellen und mit einer erhöhten Individualisierung der Anlageberatung verbunden sind, nicht aus der Luft gegriffen, da eine derartige Strategie mit hohen Investitionen verbunden ist. Auch die Dokumentation und die zugrunde liegenden Anlageberatungsprozesse müssen standardisiert und mit IT unterstützt werden, da andernfalls zu hohe (Einzelfertigungs-)Kosten entstehen. Somit stellt sich für Finanzdienstleister die Frage, ob ein Widerspruch zwischen Individualisierung der Anlageberatung und Standardisierung allgemein der Geschäftsprozesse und speziell der Dokumentation besteht und wenn ja, wie dieser gelöst werden kann.

Diese Frage betrifft das Zusammenspiel der in Abbildung 2 dargestellten Gestaltungsebenen und kann im Unternehmen nur durch eine sehr gute Abstimmung zwischen Marketing/Vertrieb und der COO-Funktion (Chief Operating Officer) auf Basis eines klar definierten Geschäftsmodells erfolgversprechend beantwortet werden. Wissenschaftlich sind solche Fragen - da interdisziplinär - anspruchsvoll und werden von der Wirtschaftsinformatik adressiert. Die „align“-Perspektive muss auf dieser Basis dafür sorgen, dass die Geschäftsprozesse hinreichend flexibel sind, um dem Wertreiber Kunde innovative Dienstleistungen wie individuelle Finanzberatungen in der erforderlichen Qualität konsistent bereitstellen zu können. Wirtschaftlich gelingt dies in den meisten Kundensegmenten nur dann, wenn die Geschäftsprozesse modular so standardisiert sind, dass die Anzahl von Prozesselementen nahe am hierfür erforderlichen Minimum liegt. 
Abbildung 2: Gestaltungsebenen der Individualisierung und Standardisierung

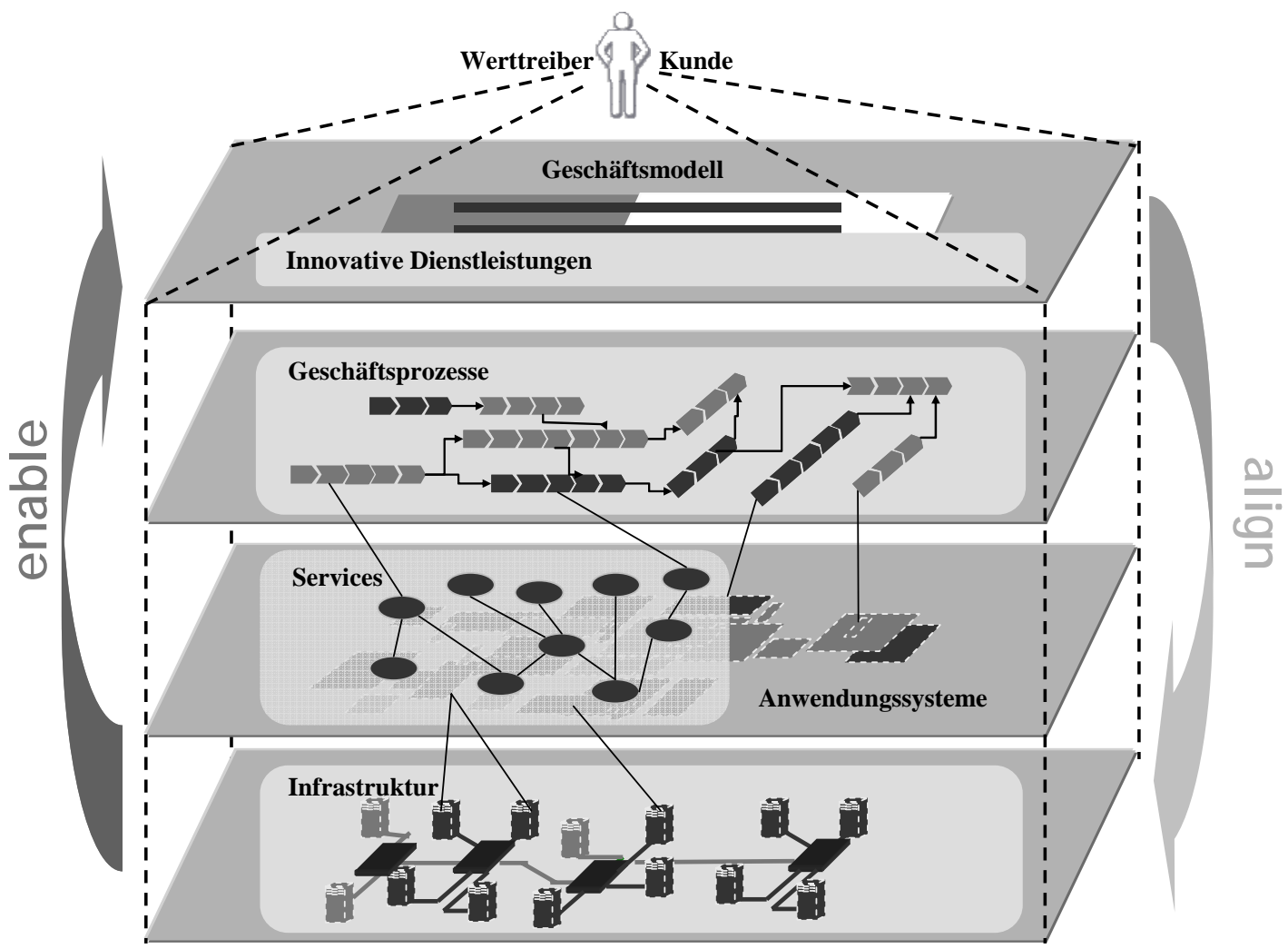

Dies erleichtert auch eine wirtschaftliche IT-Unterstützung. Durch den Einsatz möglichst standardisierter, generisch verwendbarer Services auf der Anwendungssystemebene können sowohl deren Kosten langfristig minimiert als auch die Flexibilität bei der IT-Unterstützung neuer oder veränderter Prozesse gesteigert werden. Folglich muss das Zusammenspiel mit der Infrastrukturebene so gestaltet werden, dass der sukzessive Übergang auf Service-orientierte Architekturen gelingt. Abbildung 3 illustriert dies:

Abbildung 3: Modularisierung und Standardisierung

Integration und Erweiterung durch individuelle Softwarekomponenten

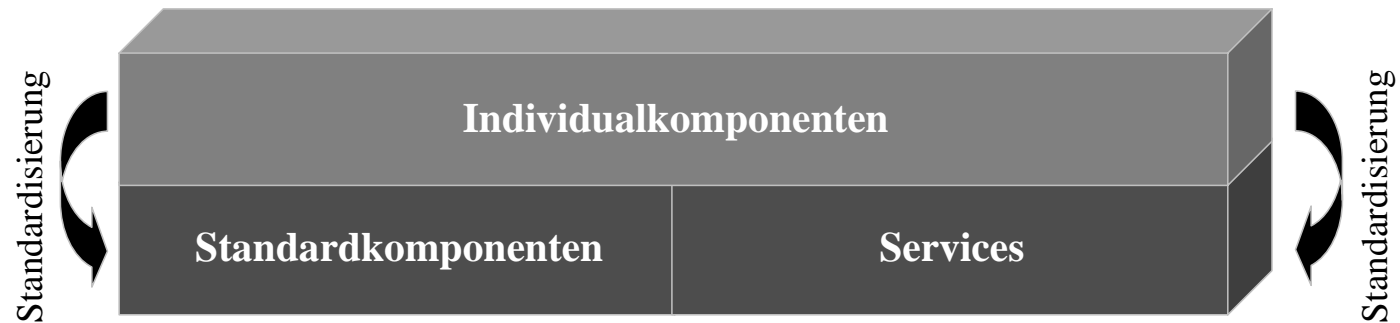

Einbindung von

Standardsoftware als Komponente (lokal verfügbar)

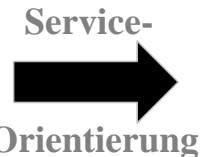

Orientierung
Einbindung von internen und externen Diensten als Service (global verfügbar) 
Umgekehrt ermöglichen in der „enable“-Perspektive technologische Fortschritte auf den unteren Ebenen sowohl kostengünstigere als auch flexiblere Prozesse und machen damit wiederum neue Dienstleistungsangebote im Interesse des Unternehmens und weiterer Kunden(segmente) wirtschaftlich. Dieses Zusammenspiel dynamisch optimal zu gestalten, ist sowohl für die Wirtschaftsinformatik als Wissenschaft als auch für die CIOs/COOs in den Unternehmen ebenso herausfordernd wie für die Unternehmen lohnenswert. Dabei zeigt sich bei richtiger Umsetzung i.d.R. in einer langfristigen Perspektive, dass zwischen Individualisierung der Anlageberatung und modularer Standardisierung von Prozessen und IT-Unterstützung kein Widerspruch besteht. Dies betrifft in unserem hier diskutierten Fall auch die Dokumentationsprozesse.

\section{V.1 Idealtypischer Ablauf einer individualisierten Anlageberatung}

Ausgangspunkt für eine individualisierte Anlageberatung ist das Kundenbedürfnis. Seine Befriedigung kann auf zwei verschiedene Weisen initiiert werden. Zum einen kann der Kunde aktiv mit seinem Bedürfnis beziehungsweise Beratungswunsch an den Berater herantreten. Der Berater sollte hier in jedem Fall das Motiv beziehungsweise die dem Beratungswunsch zugrunde liegende Situation analysieren, damit alle zweckmäßigen, eventuell vom Kunden nicht oder nicht richtig erkannten Rahmenbedingungen in der Beratung berücksichtigt werden.

Im anderen - anzustrebenden - Fall identifiziert das Finanzinstitut das Beratungspotenzial, bevor der Kunde selbst aktiv wird und das Angebot von Wettbewerbern analysiert beziehungsweise $\mathrm{zu}$ diesen abwandert. Letzteres kann beispielsweise mit Hilfe von sogenannten Referenz-Kundenlebenszyklen ${ }^{30}$ geschehen, in denen basierend auf verschiedenen Merkmalen des Kunden, seines Umfelds und seiner Historie potenzielle Beratungsanlässe ermittelt werden.

\footnotetext{
${ }^{30}$ Vgl. Heinrich, 2002.
} 
Abbildung 4: Ablauf einer individualisierten Finanzdienstleistungsberatung ${ }^{31}$

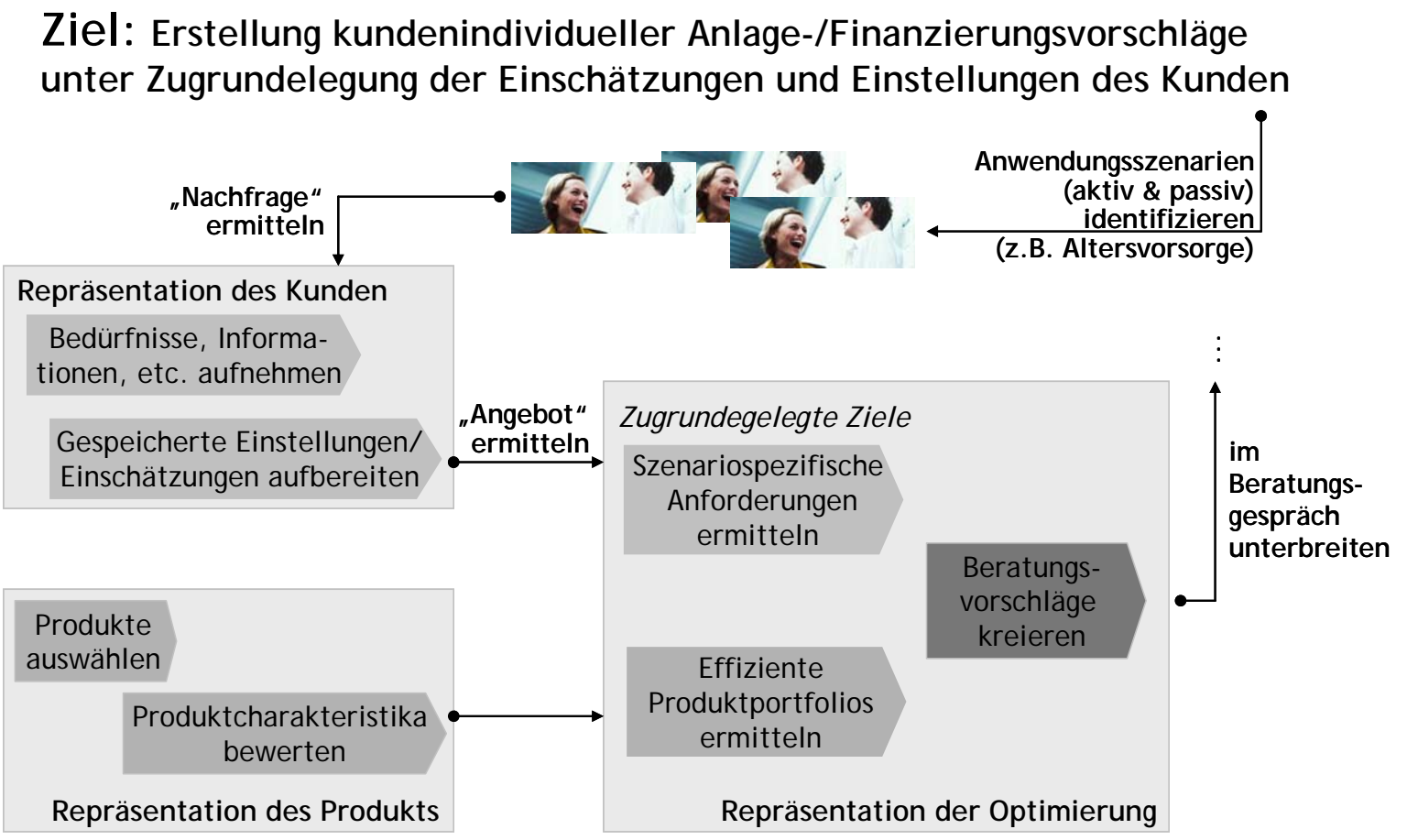

Abbildung 4 veranschaulicht einen idealtypischen Beratungsverlauf: Wurde ein Beratungsanlass aufgrund der über den Kunden gespeicherten Informationen identifiziert und konnte der Kunde für eine Beratung gewonnen werden, müssen idealtypischerweise die Kundendaten und Informationen über die Produktkategorien zur Verfügung stehen, die für die Ermittlung eines individualisierten Beratungsergebnisses notwendig sind (vergleiche Abbildung 4 „Gespeicherte Einstellungen/Einschätzungen aufbereiten“ und „Repräsentation der Produktkategorie“). Dabei lassen sich Einstellungen, welche die Bedürfnisse, Wünsche und Interessen des Kunden widerspiegeln, aus dem Verhalten des Kunden ableiten. Welches Verhalten mit welchen Kundeninformationen korreliert und welche Kundeneinstellungen aus dem Verhalten abgeleitet werden können, kann beispielsweise empirischen Studien oder der Finanzdienstleistungsliteratur entnommen werden. ${ }^{32}$ Eine Einschätzung entspricht demgegenüber dem Urteil des Finanzdienstleisters über die Grenzen und Möglichkeiten des Kunden bei seinen finanziellen Entscheidungen. Der Finanzdienstleister kann dabei seine Einschätzung über den Kunden beispielsweise aus Informationen über dessen soziale und finanzielle Situation gewinnen. Welche Einflussgrößen zu beachten sind und aus welchen Informationen sie gewonnen werden können, ist dem Wissen von Beratern, oben genannten Dokumentationspflichten und ebenfalls der Finanzdienstleistungsliteratur zu entnehmen. Die

\footnotetext{
${ }^{31}$ Siehe Buhl et al., 2004.

${ }^{32}$ Vgl. Kruschev, 1999, Tilmes, 2001, Vogelsang et al., 2000, Spiegel 2005.
} 
Einschätzungen sollten des Weiteren widerspiegeln, welche psychische Belastung dem Kunden bei Anlagen zumutbar ist. Diese ist beispielsweise aus früheren Beratungsgesprächen, aber auch seinem Agieren bei Marktveränderungen (z. B. Kurssturz bei Aktien) abzuleiten. Dabei werden Einstellungen und Einschätzungen nicht für jede Beratung neu ermittelt, sondern werden lediglich entsprechend der neu vorliegenden oder veränderten Informationen angepasst. Die Berücksichtigung der Einstellungen hat zum Ziel, das Beratungsergebnis besser auf den Kunden abzustimmen und als Folge davon die Zufriedenheit des Kunden mit der Beratung zu erhöhen. Die Beachtung der Einschätzungen soll problematische gegenwärtige oder zukünftige finanzielle Situationen des Kunden vermeiden, die durch eine Überschätzung durch den Kunden selbst oder den Berater entstehen könnten. Derartige beratungsübergreifende Kundeninformationen müssen um solche ergänzt werden, die nur auf ein Anwendungsszenario abzielen, wie beispielsweise die Höhe der Versorgungslücke im Altersvorsorgekontext (vergleiche Abbildung 4 „szenariospezifische Anforderungen ermitteln“).

Auf der Kundenseite müssen die kundenindividuelle Bedeutung der Ziele ermittelt und quantifiziert werden. Auf der Produktseite müssen die paretoeffizienten Lösungen unter Verwendung der für das jeweilige Anwendungsszenario relevanten Produktkategorien - für die Altersvorsorge beispielsweise die Kapitallebensversicherung, Rentenfonds, Immobilienfonds, Aktienfonds, private Rentenversicherung und Spareinlagen - ausgewählt werden. ${ }^{33}$ Im letzten Schritt werden aus den effizienten Produktkategorienkombinationen diejenigen ausgewählt, welche den Zielgewichtungen des Kunden am ehesten entsprechen. Für den Fall, dass die Vorschläge wider Erwarten nicht den Vorstellungen des Kunden entsprechen, das heißt die kundenindividuelle Bedeutung der Ziele durch das Beratungssystem nicht getroffen wurde, sollte der Kunde die Möglichkeit haben, selbst effiziente Beratungsvorschläge zu generieren.

\section{V.2 Konzept und prototypische Implementierung}

Die Entwicklung eines Konzepts zur individualisierten Finanzdienstleistungsberatung und dessen prototypische Umsetzung war Schwerpunkt der Forschung von vier Lehrstühlen des Forschungsverbundes FORSIP (Bayerischer Forschungsverbund für Situierung, Individualisierung und Personalisierung in der Mensch-Maschine-Interaktion).

\footnotetext{
${ }^{33}$ Vgl. Buhl et al. 2008.
} 
Abbildung 5: Konzept zur individualisierten Finanzdienstleistungsberatung

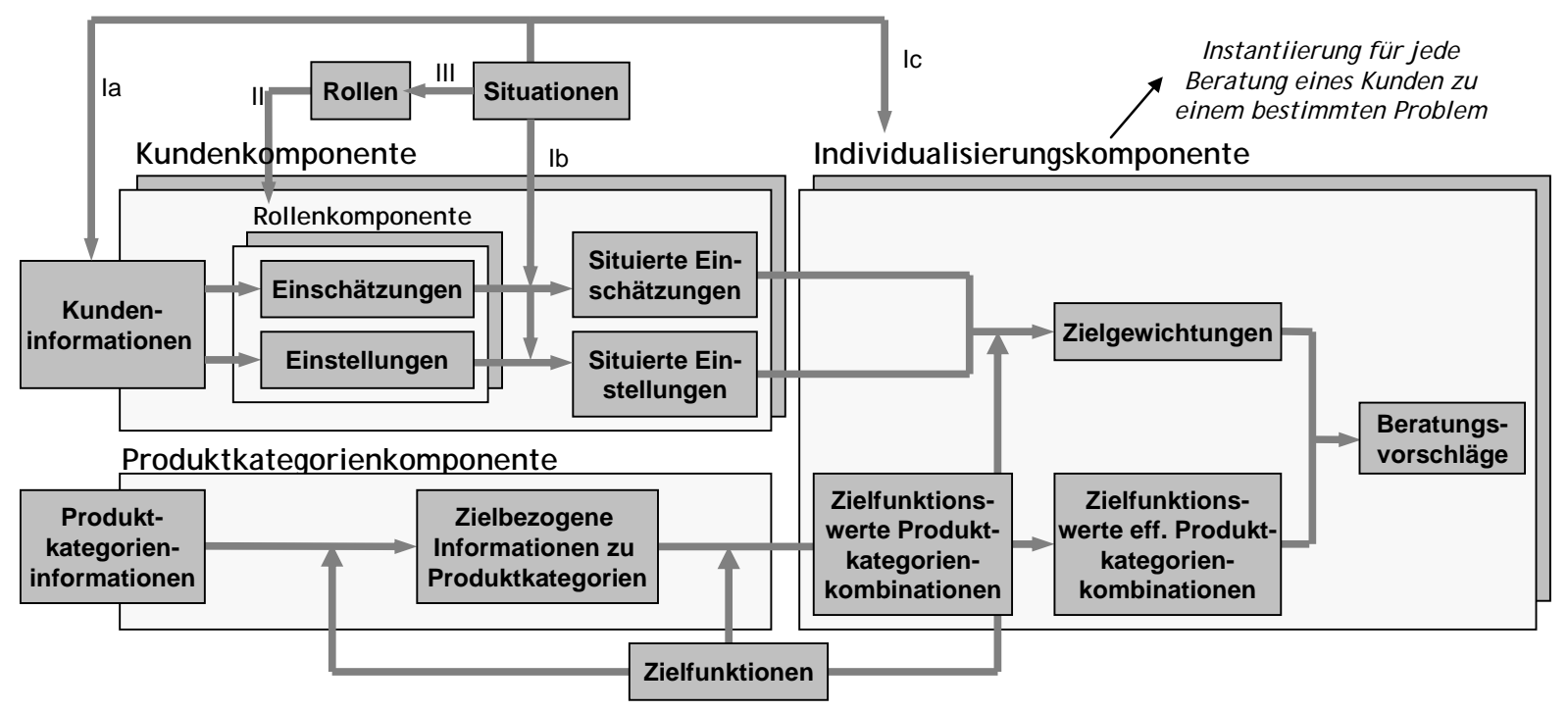

Abbildung 5 zeigt die einzelnen Schritte innerhalb des Konzepts, die ausgeführt werden um eine auf den Kunden individuell zugeschnittene Finanzdienstleistung herauszufinden. Das Konzept besteht aus der Kundenkomponente, der Produktkategorienkomponente und der Individualisierungskomponente.

Die Kundenkomponente beinhaltet das Wissen des Finanzdienstleisters über den Kunden. In ihr werden Kundendaten (z. B. Geburtsdatum, Beruf, Einkommen, Hobbies, Familienstand) verwendet, um einerseits die Einstellungen des Kunden (z. B. seine Risikoeinstellung) und andererseits die Einschätzungen des Finanzdienstleister über den Kunden (z. B. seine Risikotragfähigkeit) hinsichtlich beratungsrelevanter Aspekte abzuleiten (siehe oben). Sowohl Einstellungen als auch Einschätzungen hängen oft von der Rolle des Kunden und seiner Situation $a b$.

Die Produktkategorienkomponente enthält das notwendige Wissen über die in einer Beratung berücksichtigten Produktkategorien. Die Produktkategorienkomponente verarbeitet Daten über die Produktkategorien (z. B. historische Renditen oder Expertenschätzungen) zu zielbezogenen Informationen über Produktkategorien. Dabei wird jeder Produktkategorie ein Funktionswert gemäß ihres Beitrags für jedes der Ziele, die in der Beratung berücksichtigt werden, zugewiesen.

Die Individualisierungskomponente verwendet die Ergebnisse der beiden anderen Kategorien. Einschätzungen und Einstellungen werden in Zielgewichtungen umgewandelt, welche die Bedeutung des jeweiligen Ziels für den Kunden widerspiegeln. Darüber hinaus kombiniert die Individualisierungskomponente die Produktkategorien. Das Ergebnis sind Produktkategorienkombinationen (PKK). Im Falle einer Asset Allocation könnte eine Produktkategorienkombinationen bspw. aus 25\% Aktien(fonds), 25\% Lebensversicherungen und 50\% Renten(fonds) 
bestehen. Um die resultierenden Produktkategorienkombinationen zu bewerten, werden ihnen mit Hilfe von axiomatisch hergeleiteten Zielfunktionen Funktionswerte für jedes derjenigen Ziele zugewiesen, die in der jeweiligen Beratung berücksichtigt werden. ${ }^{34}$ Die sich ergebenden Produktkategorienkombinationen sind zum Großteil ineffizient, weswegen die dominierten aussortiert werden müssen. ${ }^{35}$ Am Ende wählt die Individualisierungskomponente aus der Menge der effizienten diejenige Produktkategorienkombination als Beratungsvorschlag aus, die den Zielgewichtungen des Kunden am besten entspricht. Dies geschieht mit Hilfe eine axiomatisch hergeleiteten Nutzenfunktion. ${ }^{36}$ Hierzu werden sowohl die Zielgewichtungen als auch die Zielfunktionswerte der effizienten Produktkategorienkombinationen verwendet.

Dieses Konzept kann innerhalb verschiedener Beratungsszenarios angewandt werden, die sich hinsichtlich der Ziele, die berücksichtigt werden, unterscheiden: Für jede Zielfunktion, die berücksichtigt wird, müssen sowohl die zielbezogenen Informationen einer Produktkategorie (Produktkategorienkomponente) als auch die Zielfunktionswerte einer Produktkategorienkombination und die Zielgewichtungen (beides in der Individualisierungskomponente) berücksichtigt werden.

\section{V.2.1 Risikovisualisierung}

Bei der Darstellung der Beratungsvorschläge nimmt die kundenverständliche Visualisierung des Risikos der Anlage eine besonders wichtige Rolle ein. Diese ist nicht nur aus Sicht des Kunden von Bedeutung, um die eigene Risikoposition einschätzen zu können. Vielmehr ist dies auch zur Vermeidung des Eintretens einer Beraterhaftung wichtig. Eine wesentliche Schwierigkeit bei der Vermittlung der Bedeutung von Risiko an den Kunden besteht darin, dass wissenschaftliche Risikomaße, wie beispielsweise die Varianz, für Kunden im Allgemeinen schwer verständlich sind. Aus diesem Grund wird der Kunde im Rahmen des FORSIP-Prototypen nicht direkt mit einem Risikomaß konfrontiert, sondern nur mit den möglichen Folgen des Eingehens von Risiko hinsichtlich seines Anlageergebnisses - im betrachteten Fall hinsichtlich der Schwankung der Höhe seiner privaten Altersrente. Dazu wird dem Kunden mittels einer grafischen Balkendarstellung der Bereich angezeigt, in dem

\footnotetext{
${ }^{34}$ Siehe hierzu Buhl et al., 2005.

${ }^{35}$ Vgl. Fielk et al., 2008.

${ }^{36}$ Vgl. Kaiser/Winkler, 2008.
} 
die Altersrente basierend auf den vorhandenen Informationen mit einer vorgegebenen Wahrscheinlichkeit liegen wird. Dabei gilt: Bei einer zu hohen Wahrscheinlichkeit leidet die Aussagekraft der Darstellung aufgrund des sich ergebenden großen Wertebereichs. Bei einer zu niedrigen Wahrscheinlichkeit ist dagegen die Gefahr groß, dass die tatsächliche monatliche Rente den angezeigten Bereich unterschreitet und sich der Kunde aus diesem Grund schlecht beraten fühlt. Um diese Problematik zu verdeutlichen, sollte dem Kunden sowohl der Wertebereich für eine Wahrscheinlichkeit von $95 \%$ als auch von $99 \%$ angezeigt werden (siehe Abbildung 2). Der Vorteil einer derartigen Darstellung liegt darin, dass dem Kunden durch den Vergleich zweier Produktkategorienkombinationen (PKK 1 und 2), die mit unterschiedlichen Risiken behaftet sind, transparent gemacht werden kann, dass die Chancen steigender Risikobereitschaft gleichzeitig mit den Risiken höherer Verluste verbunden sind. Gleichzeitig wird das zunächst abstrakte Risikomaß durch die gewählte Darstellung konkrete Euro-Beträge - für den Kunden in seinen möglichen Folgen verständlich. Die Auswirkungen einer geringeren monatlichen Rente als die erwartete, lassen sich direkt auf dann nötige Einsparungen im täglichen Leben übertragen. Des Weiteren ist für den Kunden deutlich erkennbar, dass, je höher die Wahrscheinlichkeit gewählt wird, sich der mögliche Wertebereich stark vergrößert und damit die Aussagekraft der Darstellung sinkt.

Abbildung 6: Visualisierung des Wertebereichs für die erwartete monatliche Rente im Rahmen einer individualisierten Altersvorsorgeberatung

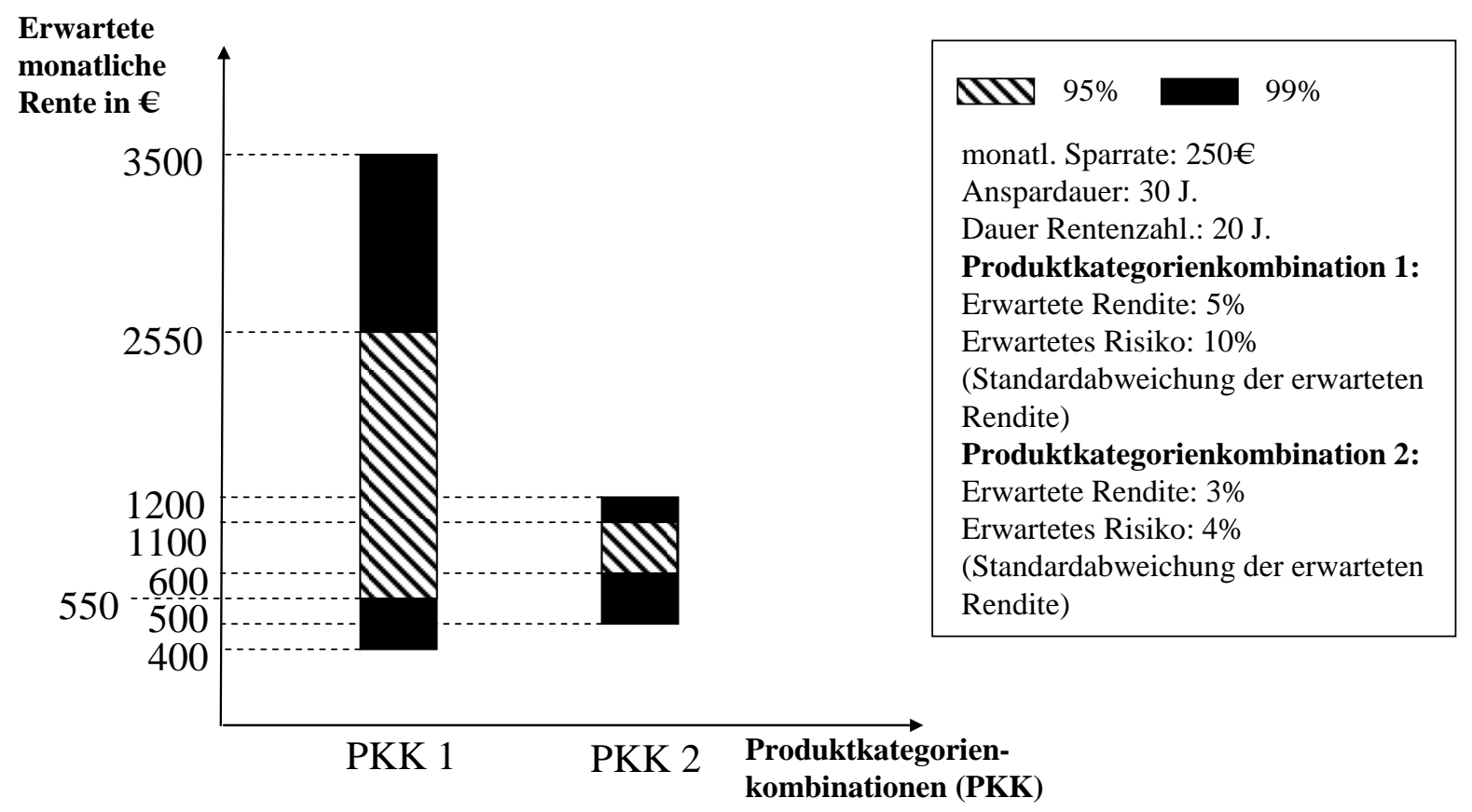

Der Nachteil der gewählten Darstellung liegt darin, dass die tatsächlich erreichte Rente auch außerhalb des dargestellten Bereichs liegen kann. Gleichzeitig wird hier nicht angegeben, wie 
stark eine Abweichung ausfällt, wenn die Rendite außerhalb des 99 \%-Bereichs liegt. Um auch hier dem Thema Beratungshaftung gerecht zu werden, ist es wichtig, dass dies dem Kunden deutlich kommuniziert wird.

\section{V.2.2 Visualisierung der Zielfunktionswerte eines Beratungsergebnisses}

Bei der Ermittlung eines individualisierten Beratungsergebnisses reicht es jedoch nicht aus, nur die quantitativen Ziele Rendite und Risiko zu betrachten. Vielmehr kommt die einschlägige Literatur zu dem Schluss, dass gerade im Bereich der Anlageberatung auch eher qualitative, d.h. schwer quantifizierbare Ziele berücksichtigt werden müssen: ${ }^{37}$

- Liquidierbarkeit (bezeichnet die Möglichkeit, Produktkategorien jederzeit zu fairen Preisen verkaufen zu können)

- Variabilität (bemisst den Ausmaß, in dem ein Kunde bestimmte Parameter einer Produktkategorienkombination während der Laufzeit ändern kann, z. B. die Höhe oder die Häufigkeit einer Sparrate)

- Nachvollziehbarkeit (bildet das Bestreben eines Kunden ab, die Transaktionen zwischen den beteiligten Akteuren und die Folgen nach dem Erwerb einer bestimmten Produktkategorienkombination zu verstehen)

- Verwaltbarkeit (repräsentiert den Wunsch eines Kunden, den Aufwand für die Verwaltung einer Produktkategorienkombination während der Laufzeit zu minimieren)

Eine besondere Herausforderung der Beratung ist, dem Kunden die Interdependenz der Ziele verständlich zu kommunizieren. Insofern soll eine einfache Darstellung verwendet werden, um den Zusammenhang zwischen der Ausprägung der gewählten Ziele und der Zusammensetzung des Beratungsergebnisses deutlich zu machen. Darüber hinaus soll dem Kunden mittels einer einfachen, für ihn nachvollziehbaren Funktionalität des IT-gestützten Beratungssystems die Möglichkeit gegeben werden, die Zielausprägungen zu variieren und die Auswirkung auf die anderen Ziele zu beobachten. Dies wurde im Rahmen des FORSIPPrototyps mittels einfacher Schieberegler visualisiert, wie in Abbildung 7 dargestellt.

\footnotetext{
${ }^{37}$ Vgl. Lease et al., 1976, Buhl et al., 2008, Spiegel, 2005.
} 
Abbildung 7: Visualisierung der Zielfunktionswerte des Beratungsergebnisses im Rahmen einer individualisierten Altersvorsorgeberatung
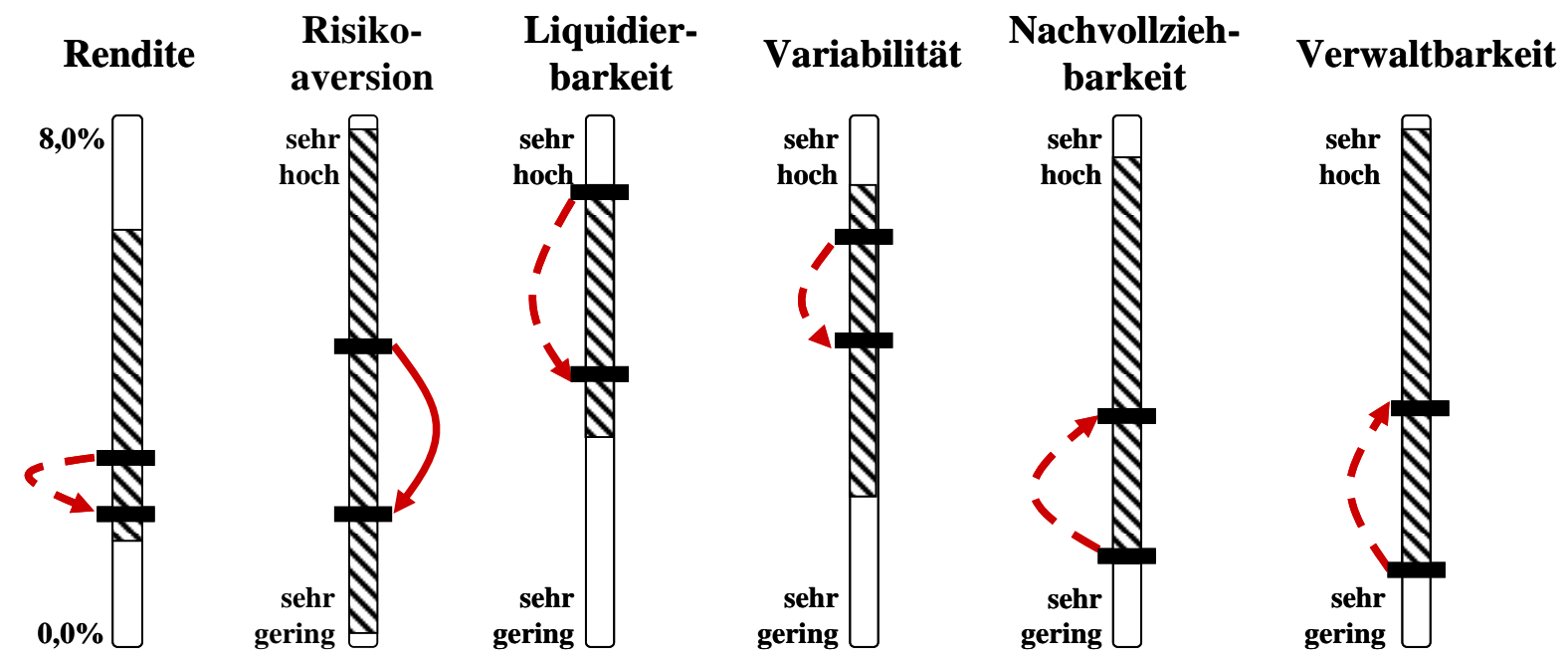

Um dem Kunden für ihn verständliche Maßeinheiten für die Skalen zu geben, wurden die Zielausprägungen in nachvollziehbare Größen übersetzt. Während die Angabe einer prozentualen Rendite für das Renditeziel für den Kunden im Allgemeinen direkt verständlich sein dürfte, ist es bei den anderen Zielen erforderlich, eine Übersetzung der tatsächlichen Ausprägungen in die Maßeinheiten „sehr hoch“ bis „sehr niedrig“ vorzunehmen. Die schwarzen Balken am Ausgangspunkt der Pfeile visualisieren dabei die Zielfunktionswerte einer vom Beratungssystem empfohlenen kundenindividuellen Produktkategorienkombination. Wird einer der Balken durch den Kunden in seiner Position (vergleiche durchgezogener Pfeil in Abbildung 7) verändert, da der Kunde beispielsweise das Risiko des ermittelten Vorschlags als zu hoch empfindet, wird aus der Menge der effizienten Produktkategorienkombinationen eine ausgewählt, deren Zielfunktionswert hinsichtlich des veränderten Ziels dem durch den Kunden bestimmten entspricht. Als Folge werden auch die Balken der anderen Regler gemäß den Zielfunktionswerten der neu gewählten Produktkategorienkombination verschoben. Dem Kunden wird dadurch visualisiert, dass die Veränderung eines Ziels immer eine Veränderung der Werte der anderen Ziele nach sich zieht. Basis für die Auswahl neuer Produktkategorienkombinationen sind dabei immer die ermittelten effizienten Produktkategorienkombinationen. Da nicht für den gesamten Wertebereich der Schieberegler effiziente Produktkategorienkombinationen vorliegen, wird durch die schraffierte Fläche der Bereich verdeutlicht, in dem die Schieberegler verschoben werden können, das heißt angesichts der aktuell vorliegenden Daten Beratungsvorschläge gefunden werden können. 


\section{V.2.3 Nutzung von Situationen zur Simulation von Szenarien}

Das FORSIP-Konzept berücksichtigt die konkrete Situation des Kunden sowie seine Rolle zum Zeitpunkt der Beratung, da beide Konstrukte Einfluss auf die Einschätzungen und Einstellungen haben (siehe Abbildung 7). ${ }^{38}$ Das Wissen über die Zusammenhänge zwischen bestimmten Situationen und deren Auswirkungen auf die Anlage lässt sich daher auch nutzen, um dem Kunden die Entwicklung des Anlagebetrags oder die Veränderung der Portfoliozusammensetzung zu simulieren und anhand von Grafiken zu veranschaulichen, wenn eine zukünftig geplante oder unerwünschte Lebenssituationen eintritt oder sich die Umweltsituation verändert. Damit können dem Kunden sehr viel robustere Anlagevorschläge unterbreitet werden, die beispielsweise bei Arbeitslosigkeit oder einer Scheidung nicht zu prohibitiven Transaktionskosten führen.

Eine Veränderung der Portfoliozusammensetzung kann beispielsweise anhand von Tortendiagrammen dargestellt werden. So zeigt der folgende Screenshot aus dem im Rahmen von FORSIP entwickelten Prototypen zur Altersvorsorgeberatung, wie sich die Portfoliozusammensetzung verändern würde, wenn der Kunde heiraten und anschließend Kinder bekommen würde.

Abbildung 8: Veränderung der Portfoliozusammensetzung in Abhängigkeit zukünftiger geplanter Situationen

Geben Sie an, wie Sie Ihr Leben planen und es werden Ihnen die notwendigen Umschichtungen angezeigt!

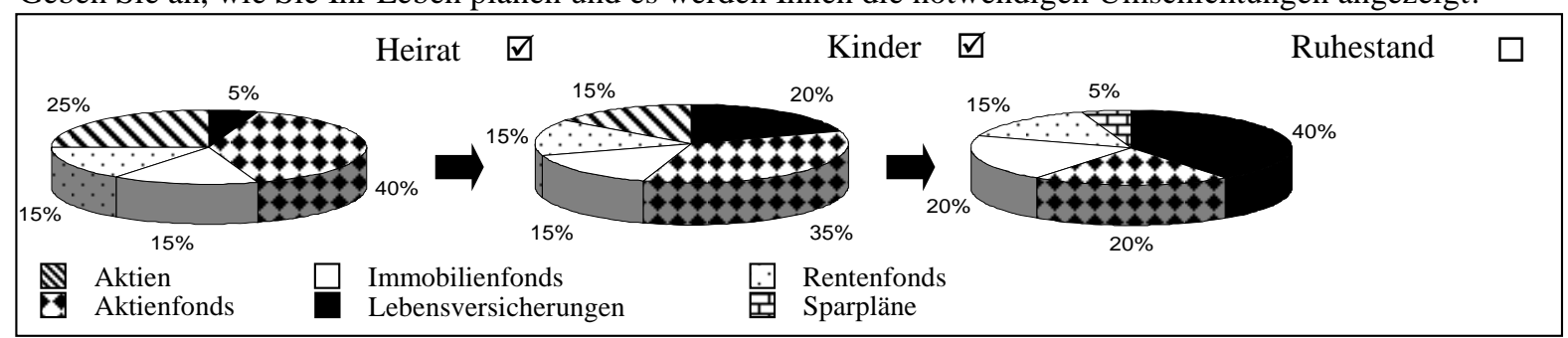

Die Veränderung der Entwicklung des Anlagebetrags bei Eintreten einer bestimmten Situation kann veranschaulicht werden, indem beispielsweise der bisher erwartete dem aufgrund der Situation (positiv oder negativ) veränderten Auszahlungsbetrag der Anlage zum Ende der Laufzeit gegenübergestellt wird. So kann im FORSIP-Prototypen ausgewählt werden, welche Situation (Arbeitslosigkeit, Berufsunfähigkeit oder Inflation) simuliert werden soll, wobei jeweils angegeben werden muss, wie und für welchen Zeitraum sich die mögliche Sparrate oder der Zinssatz verändern. In der Grafik werden die sich ergebenden

\footnotetext{
${ }^{38}$ Vgl. auch Buhl et al., 2007.
} 
erwarteten monatlichen Rentenbeträge ohne und mit Berücksichtigung der Situation gegenübergestellt.

Abbildung 9: Einfluss von unerwarteten Situationen auf die Entwicklung des Portfolios

In der folgenden Grafik sollen Ihnen die Auswirkungen von unerwarteten Situationen auf Ihre Altersvorsorge veranschaulicht werden.

\begin{tabular}{|ll|}
\hline \multicolumn{2}{|l|}{ Wählen Sie bitte die zu } \\
betrachtende Situation aus: \\
Arbeitslosigkeit & $\odot$ \\
Berufsunfähigkeit & $\bigcirc$ \\
Inflation & \\
Geben Sie die veränderte Sparrate und \\
die Dauer der Änderung an: \\
Sparrate (in €) & 100 \\
Beginn & 2010 \\
Dauer (Jahre) & 5 \\
\hline
\end{tabular}

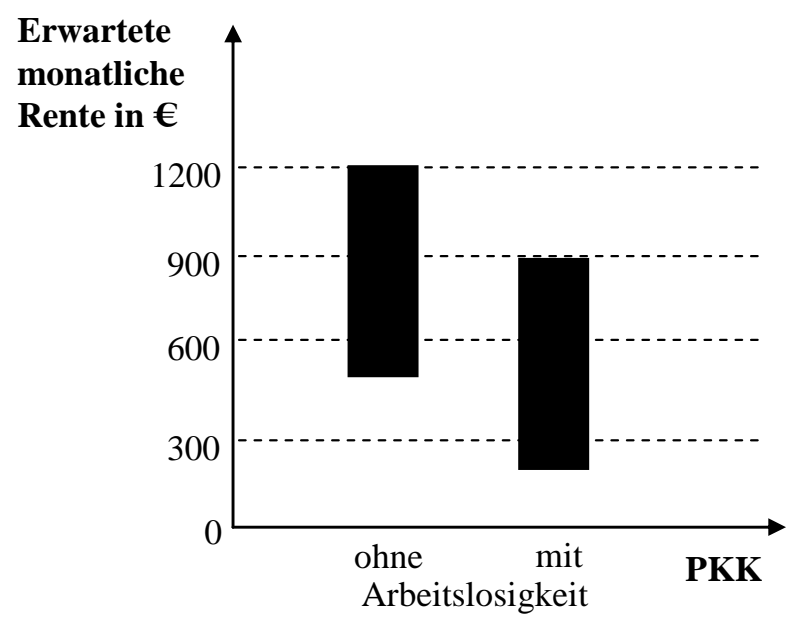

Zusammenfassend lässt sich damit sagen, dass Situationen im Rahmen einer Beratung für viele Aspekte genutzt werden können. Neben der hier geschilderten Nutzung, dem Kunden ein Gefühl für die Auswirkung - insbesondere deren Ausmaß - bestimmter künftiger Situationen geben können, werden Situationen auch zur Ableitung von Beratungsanlässen verwendet. Darüber hinaus nehmen sie Einfluss auf die grafische Aufbereitung und den Detaillierungsgrad der Informationen bei der Beratung und die Auswahl des Beratungsergebnisses.

\section{V.3 Transfer in die Praxis}

Die Ergebnisse des FORSIP-Projekts stellen eine wesentliche Verbesserung gegenüber den heutzutage in der Praxis eingesetzten Lösungen dar. So berücksichtigt das Konzept auch Steuern, Transaktionskosten und das sogenannte Background Risk, ${ }^{39}$ welche in der Praxis häufig vernachlässigt werden. Zudem werden in der FORSIP-Lösung ausschließlich hinsichtlich der genannten Ziele effiziente Vorschläge ermittelt, was am Markt häufig nicht sichergestellt ist. Ein weiteres wesentliches Ergebnis des Projekts ist die Veranschaulichung der Wechselwirkungen zwischen den Zielen.

\footnotetext{
${ }^{39}$ Siehe Cvitanic et al., 2007 und Klos et al., 2003.
} 
Abbildung 10: Übertragung der FORSIP-Ergebnisse in die Praxis

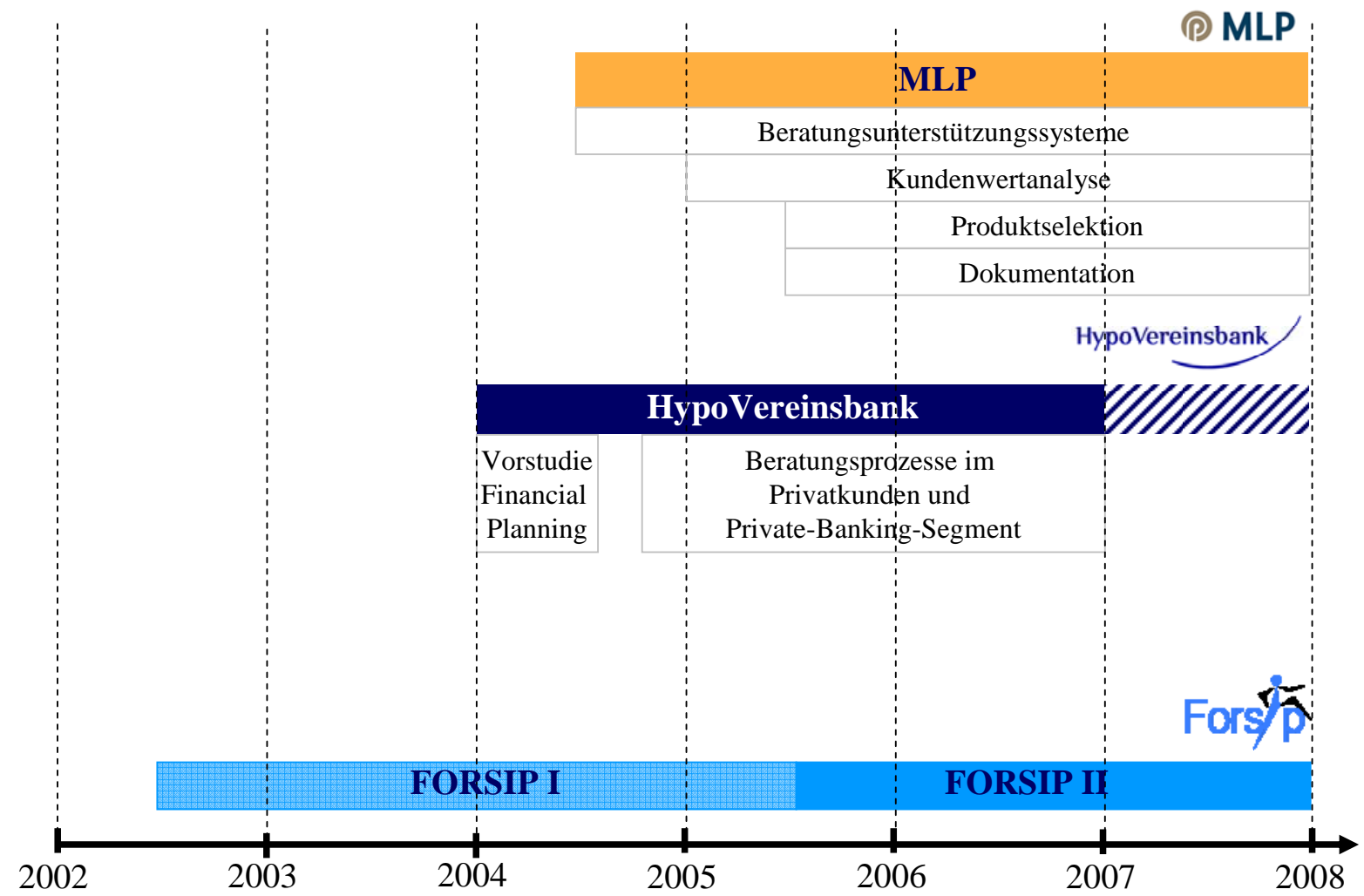

Diese - in zwei Phasen eines aus öffentlichen Mitteln geförderten Projekts erarbeiteten Ergebnisse mit Grundlagencharakter wurden sukzessive auch in Kooperationen mit Praxispartnern aus dem Finanzdienstleistungsbereich eingesetzt und trugen dort dazu bei, eine individualisierte Anlageberatung anzubieten (siehe Abbildung 10). Dieser Transfer öffentlich geförderter Forschungsergebnisse in die Praxis hat sich bei Projekten des Kernkompetenzzentrums Finanz- und Informationsmanagement an der Universität Augsburg bereits mehrfach bewährt.

\section{Literaturübersicht}

Arbeitskreis EU-Vermittlerrichtlinie Dokumentation, 2007, Hausrat Risikoanalyse, http://www.vermittlerprotokoll.de/download/hausrat/hausrat risikoanalyse.doc, Abruf am 01.11.2007.

Balzer, P., 2007, Umsetzung der MiFID: Ein neuer Rechtsrahmen für die Anlageberatung, in: Zeitschrift für Bankrecht und Bankwirtschaft, 19. Jg., S. 333-345.

Böckhoff, M./Stracke, G., 2003, Der Finanzplaner: Handbuch der privaten Finanzplanung und individuellen Finanzberatung. Heidelberg. 
Booz Allen Hamilton GmbH, 2007, Booz Allen Hamilton-Studie: Deutsche Geschäftsbanken verharren im Mittelmaß - Nur jeder Dritte ist mit der Betreuung in der Filiale zufrieden, http://www.boozallen.de/presse/pressemitteilungen/pressemitteilung-detail/38004436, Abruf am 21.12.2007.

Buhl, H.U./Kundisch, D./Steck, W., 2002, Sophistication Banking als erfolgreiche Strategie im Informationszeitalter, in: Zeitschrift für Betriebswirtschaft, 72. Jg., Ergänzungsheft 2, S. 1-12.

Buhl, H.U./Heinrich, B./Steck, W./Winkler, V., 2004, Konzept zur individualisierten Finanzdienstleistungsberatung für Privatkunden und dessen prototypische Umsetzung, in: Wirtschaftsinformatik, 46. Jg., S. 427-438.

Buhl, H.U./Volkert, S./Winkler, V., 2005, Individualisierte Anlageberatung: Axiomatische Fundierung von Zielfunktionen zur Bewertung von Anlagealternativen, in: Ferstl, O./Sinz, E./Eckert, S./Isselhorst, T. (Hrsg.), Wirtschaftsinformatik 2005 - eEconomy eGovernment eSociety, Heidelberg, S. 489-508.

Buhl, H.U./Kaiser, M./Winkler, V., 2007, Beratungsindividualisierung in der Finanzdienstleistungsbranche - Umsetzungskonzepte und rechtliche Rahmenbedingungen, in: Wirtschaftsinformatik, 49. Jg., S. 26-33.

Buhl, H.U./Fridgen, M./Kaiser, M., 2008, Konzepte für die Abbildung „harter“ und „weicher“ Ziele in der individuellen Altersvorsorgeberatung, angenommener Beitrag für:

Multikonferenz Wirtschaftsinformatik 2008, München.

Bund der Versicherten, 2007, Hausrat - Bedarf, http://www.bundderversicherten.de/bdv/default.asp?ContentUrl=http://www.bundderversiche rten.de/bdv/Versicherungsarten/Hausrat/bedarfhausrat.htm\&TopUrl=/bdv/navi/headline_sho w.asp?headline=Bedarf, Abruf am 01.11.2007.

Bundesverband Deutscher Banken, 2007, Gut beraten - Neun von zehn Kunden sind mit ihrer Bank zufrieden, http://www.bankenverband.de/channel/111010/art/2097/index.html, Abruf am 26.09.2007.

Cvitanic, J./Goukasian, L./Zapatero, F., 2007, Optimal Risk Taking with Flexible Income, in: Management Science, 53. Jg., S. 1594-1603.

Diegelmann, M./Deter, H., 2007, E.B.I.F. sets the pace for customer orientation - Great demand for the latest CRM solutions, http://ebif.com/en/press/pressreleases/2006/20061023.shtml, Abruf am 03.11.2007.

Duve, C./Keller, M., 2006, MiFID: Die neue Welt des Wertpapiergeschäfts oder: Glauben Sie nicht, Sie könnten ohne weiteres Wertpapiere kaufen, in: Betriebsberater, 61. Jg., S. 24772484.

Fiala, J., 2007, Nur wer gut berät, ist gut geschützt, in: versicherungsmagazin, 54. Jg., S. 6465.

Fielk, G./Kaiser, M./Winkler, V., 2008, Approximating efficient portfolios - An evolutionary algorithm for a multi-objective problem, Diskussionspapier des Lehrstuhls WI-IF, in Begutachtung. 
Fridgen, M./Steck, W., 2002, Customer Tracking in the Internet: New Perspectives on Web Site Controlling, in: Quarterly Journal of Electronic Commerce, 3. Jg., S. 235-245.

Harrison, D., 2005, Personal Financial Planning: Theory and Practice, Harlow.

Hedley, K./White, J./Petit dit de la Roche, Cormac/Banerjea, S., 2006, Banking 2015: a classic strategy battle of scale vs focus, in: Strategy \& Leadership, 34. Jg., S. 51-58.

Heinrich, B./Kaiser, M./Klier, M., 2008, Does the EU Insurance Mediation Directive help to improve Data Quality - a metric-based approach, Diskussionspapier des Lehrstuhls WI-IF, in Begutachtung.

Heinrich, B., 2002, Methode zur wertorientierten Analyse und Gestaltung der Kundenbeziehung - Zur Rolle des Service Integrators im Privatkundengeschäft von Kreditinstituten, Berlin.

Hetzer, J./Palan, D., 2007, Bankentest - Vernichtendes Urteil, http://www.managermagazin.de/geld/geldanlage/0,2828,489764,00.html, Abruf am 21.12.2007.

Jagersma, P.K., 2006, Strategic marketing and the global banking industry: elements of excellence, in: Journal of Business Strategy, 27. Jg., S. 50-59.

Kaas, K.P./Severidt, K., 2002, Neue Preismodelle in der Kapitalanlageberatung der Banken? in: Zeitschrift für Betriebswirtschaft, 72. Jg., S. 619-640.

Kaiser, M./Winkler, V., 2008, Axiomatic considerations for an individualised multi-objective advisory in the financial services sector, Diskussionspapier des Lehrstuhls WI-IF, in Begutachtung.

Klöckner, B.W., 2007, Peinliche Ergebnisse - große Chancen, in: Bankmagazin, 55. Jg., S. 4243.

Klos, A./Langer, T./Weber, M., 2003, Über kurz oder lang - Welche Rolle spielt der Anlagehorizont bei der Beurteilung von Investments?, in: Zeitschrift für Betriebswirtschaft, 73. Jg., S. 733-765.

Kruschev, W., 1999, Private Finanzplanung: Die neue Dienstleistung für anspruchsvolle Anleger, Wiesbaden.

Kundisch, D., 2003, New Strategies for Financial Services Firms: The Life-Cycle-Solution Approach, Heidelberg.

Lease, R.C./Lewellen, W. G./Schlarbaum, G. G., 1976, Market Segmentation: Evidence on the Individual Investor, in: Financial Analysts Journal, 32. Jg., S. 53-60.

Maschmeyer, C., 2007, Vermittlerrichtlinie: Unabhängige als Gewinner, in: bank und markt, 36. Jg., S. 16-18.

Mertens, P./Griese, J., 2002, Integrierte Informationsverarbeitung, Band 2: Planungs- und Kontrollsysteme in der Industrie, Wiesbaden. 
Nellis, J.G./McCaffery, K. M./Hutchinson, R. W., 2000, Strategic challenges for the European banking industry in the new millenium, in: International Journal of Bank Marketing, 18. Jg., S. 53-63.

Oberhuber, N., 2007, Beratung nach Schema F, http://www.zeit.de/2007/49/GS-Aufmacher, Abruf am 2007/12/06.

Porter, M., 1998, Competitive advantage - creating and sustaining superior performance, New York.

Roßnagel, A., 2005, Verantwortung für Datenschutz, in: Informatik-Spektrum, 28. Jg., S. 462473.

Schmidt, G., 2005, Persönliche Finanzplanung: Modelle und Methoden des Financial Planning, Berlin.

Schmidt-Gallas, Dirk; Lauszus, Dieter; Burger, Verena (2007): Versicherungsvertrieb im Umbruch.

Shapiro, C./Varian, H.R., 1998, Information Rules - A Strategic Guide to the Network Economy, Boston.

Sherwood, T.J., 1995, Executive forum: Private Banking in Europe, in: International Journal of Bank Marketing, 13. Jg., S. 29-35.

Spiegel (2005): Debit and Credit 6. In: TNS; MMA; Sinus Sociovision; ISBA (Hrsg.): Spiegel, Hamburg

Steevens, C., 2007, "Verbünde haben es schwerer als Konzerne" - Interview mit Karl-Peter Schackmann-Fallis vom DSGV, in: Börsen-Zeitung, S. 5.

Stiftung Warentest, 2007, Beraten und verkauft, in: Finanztest, 17. Jg., S. 12-16.

Stiftung Warentest, 2007, Versicherungsvermittlung - Etwas mehr Sicherheit für Kunden, http://www.stiftungwarentest.de/online/versicherung_vorsorge/meldung/1447512/1447512.html, Abruf am 01.11.2007.

Tilmes, R., 2001, Financial Planning im Private Banking - Kundenorientierte Gestaltung einer Beratungsdienstleistung, Bad Soden/Ts.

Vogelsang, D./Sachs, P./Uppena, J.M./Oehme, M./Liebing, R./Knorr, K., 2000, Handbuch Finanz- und Vermögensgestaltungsberatung - Neues Geschäftsfeld für steuer- und rechtsberatende Berufe, Stuttgart.

Warth, W.P./Beenken, M., 2005, Bankassurance nach EU-Recht: Abschied vom Produktvertrieb, in: bank und markt, 55. Jg., S. 36-38.

Yildirim, N., 2004, Datenschutz im Electronic Government - Risiken, Anforderungen und Gestaltungsmöglichkeiten für ein datenschutzgerechtes und rechtsverbindliches eGovernment, Wiesbaden. 